\title{
Earthshine Observations of the Earth's Reflectance
}

\author{
P. R. Goode, ${ }^{1}$ J. Qiu, V. Yurchyshyn, J. Hickey \\ Big Bear Solar Observatory, New Jersey Institute of Technology, Newark, NJ 07102
}

M-C. Chu

Department of Physics, The Chinese University of Hong Kong, Shatin N.T., Hong Kong

E. Kolbe

Departement für Physik and Astronomie, Universität Basel, Basel, Switzerland

C. T. Brown, S. E. Koonin

W. K. Kellogg Radiation Laboratory, California Institute of Technology, Pasadena, CA 91125

\begin{abstract}
Regular photometric observations of the moon's "ashen light" (earthshine) from the Big Bear Solar Observatory (BBSO) since December 1998 have quantified the earth's optical reflectance. We find large $(\sim 5 \%)$ daily variations in the reflectance due to large-scale weather changes on the other side of the globe. Separately, we find comparable hourly variations during the course of many nights as the earth's rotation changes that portion of the earth in view. Our data imply an average terrestrial albedo of $0.297 \pm 0.005$, which agrees with that from simulations based upon both changing snow and ice cover and satellite-derived cloud cover $(0.296 \pm 0.002)$. However, we find seasonal variations roughly twice those of the simulation, with the earth being brightest in the spring. Our results suggest that long-term earthshine observations are a useful monitor of the earth's albedo. Comparison with more limited earthshine observations during 1994-1995 show a marginally higher albedo then.
\end{abstract}

A central parameter of the earth's climate is the planet's albedo, $A$, the fraction (roughly 0.3 ) of the incident sunlight that it reflects. Simple power balance considerations suggest the importance of this parameter: in the absence of feedbacks, the earth's blackbody temperature of some $255 \mathrm{~K}$ would increase by roughly $1 \mathrm{~K}$ for a 0.01 decrease in $A$. Precise measurement of the albedo can reveal variation with the earth's aspect (land is more reflective than water), weather (cloud patterns), and seasons (snow and ice cover and vegetation).

The albedo is determined most directly by satellite observations [Gibson et al., 1990; Stephens, Campbell and Vonder Haar, 1981; Rossow and Lacis, 1990]. However, satellites in

\footnotetext{
${ }^{1}$ Also at W. K. Kellogg Radiation Laboratory, California Institute of Technology, Pasadena, CA 91125
}

Copyright 2001 by the American Geophysical Union. low-earth orbit view only a small fraction of the earth's surface at once so that many observations must be combined to obtain global-scale information. It is also a major technical and logistical challenge to keep calibrated photometers continuously in orbit on decadal timescales.

The earthshine, or "ashen light", is the glow of the "dark" part of the lunar disk visible to a nighttime observer. It is sunlight reflected from the earth and retroflected from the lunar surface, and so offers an alternative route to studying the earth's reflectance. Earthshine data are complementary to existing satellite data in that the coverage is instantaneous and hemispheric in scale. Because the earth's phase as seen from the moon is supplementary to that of the moon seen from the earth (i.e., the earth is nearly "full" when the moon is a thin crescent), the instantaneous intensity of the earthshine near the new moon samples almost half of the earth. For over a quarter century, beginning in 1926, Danjon and his followers [Danjon, 1928, 1954; Dubois, 1947] performed regular earthshine observations from France. We have reinvigorated and modernized this nearly forgotten way of measuring the earth's albedo.

Our earthshine coronagraph is a 6 -inch refractive telescope with a cooled AP-7 $512 \times 512$ pixel CCD camera; our fiducial regions comprise about 100 pixels each. We mask the bright portion of the lunar disk (i.e., "moonshine") with a neutral density filter of transmission $\sim 10^{-5}$, and correct the measured earthshine intensity for the effects of moonshine scattered in the atmosphere and optical train. For details, see http://ww .bbso.njit. edu/Research/Earthshine.

The earthshine intensity is most conveniently determined by observing the specific intensities $I_{a, b}$ of two nearly diametrically opposite lunar highland regions (each covering nearly $0.1 \%$ of the lunar disk) near the edge of the disk one $(a)$ in the earthshine, the other $(b)$ in the moonshine. If we define the earth's effective albedo, $A^{*}$, as that of a Lambert (perfectly diffusing) sphere having the same reflectance [Flatté, Koonin and MacDonald, 1992], then

$$
A^{*}=\frac{3}{2} \frac{1}{f_{L}(\beta)}\left[\frac{R_{e m}}{R_{e}}\right]^{2}\left[\frac{R_{o a}}{R_{o b}}\right]^{2}\left[\frac{R_{e s}}{R_{m s}}\right]^{2} \frac{p_{b} f_{b}(\theta)}{p_{a} f_{a}(\gamma)} \frac{I_{a}}{I_{b}} .
$$


Here, $\theta$ is the lunar phase ( $\theta$ is nearly $-\pi$ at the new moon, increases to about $\theta=0$ at mid-month and is nearly $\theta=\pi$ at the end of the lunar month), $f_{b}(\theta)$ is the phase function of region $b, \beta \approx \pi-\theta$ is the earth's phase angle, and $f_{L}(\beta)=$ $[(\pi-|\beta|) \cos \beta+\sin |\beta|] / \pi$ is the Lambert phase function. (All phase functions are defined so that $f(0)=1$.) The lunar phase function, $f_{a}(\gamma)$, arises from the near retroflection of the earthshine by the moon, where $\gamma \sim^{\circ}$ is the angle between the incident and nearly retroflected earthshine from region a. $R_{e m}, R_{e}, R_{o a}, R_{o b}, R_{e s}$ and $R_{m s}$ are the earth-moon distance, the radius of the earth, the distance of the observer from patches $a$ and $b$, the earth-sun distance and the moonsun distance, respectively.

Our differential measurement of the earthshine removes many of the complications associated with varying atmospheric absorption and solar intensity. Lunar images around a lunar eclipse determine the ratio of the geometrical albedos of the two fiducial regions, $p_{b} / p_{a}$, while photometric observations during many lunations determine the phase functions $f_{a, b}$.

To fully account for the effect of atmospheric extinction, observations of both the earthshine and the moonshine are made as the airmass varies through the night. The true intensities to be used in eqn. (1) are greater than the observed intensities by a factor of $e^{\alpha z}$. The local airmass, $z$, is the cosecant of the elevation angle for observations near the zenith, while near the horizon the curvature of the earth requires that we use tabulated values [Kondratev, 1973]. The atmospheric extinction coefficient, $\alpha$, ranges between about 0.1 and 0.3 , and on a typical night it is determined with a relative precision of $1 \%$ for the earthshine and about $0.5 \%$ for the moonshine by fitting that night's observations. Typically, we find $\alpha_{e}-\alpha_{m} \sim 0.05$.

To determine the earth's reflectance from the earthshine, we must know the lunar phase functions, $f_{a, b}$ - the moonshine intensity of the fiducial regions as a function of the lunar phase. However, raw moonshine data near the quarter moon show as much as a $5 \%$ variation from month-to-month at the same lunar phase due to varying atmospheric absorption, the lunar declination, and libration. We have addressed this problem by making three successive, linear adjustments to the observed moonshine intensities using the integrated crescent of the moon as a standard, and then correcting for the declination and libration of the moon. After applying these three physically required corrections, the aforementioned $5 \%$ variation in $f_{a, b}$ is reduced to $0.5 \%$. With these adjustments, we estimate that we can measure the nightly effective albedo to a relative precision of about $1 \%$ on clear nights. This precision is comparable to those quoted for satellite albedo data [Gibson et al., 1990; Stephens, Campbell and Vonder Haar, 1981; Rossow and Lacis, 1990].

Our results are best interpreted by comparing them to simulations. In modeling the reflectance of the earth, we use scene models developed for the Earth Radiation Budget Experiment (ERBE) observations [Suttles et al., 1988]. The cloud cover data, updated every 6 hours, are a whole earth composite of visible-light satellite images from WSIINTELLICAST (http://www.intellicast.com/). For the snow and ice cover information, we use simulations from the Canadian Center for Climate Modeling and Analysis (http://www.cccma.bc.ec.gc.ca), which gives the monthly mean snow/ice cover for each computational cell $\left(2.5^{\circ} \times\right.$ $\left.2.5^{\circ}\right)$.

Figure 1 shows the observed (left panel) and simulated (right panel) effective albedo expressed in astronomical magnitudes, $\left[A^{*}\right] \equiv-2.5 \log A^{*}$, as determined from our earthshine observations. This standard astronomical definition implies that the larger $\left[A^{*}\right]$ is, the smaller the albedo. Note that $\left[A^{*}\right]=1.31$ for $A^{*}=0.3$ and that a $1 \%$ change in $A^{*}$ corresponds very nearly to a 0.01 change in $\left[A^{*}\right]$. The $A^{*}$ 's from morning and evening observations and simulations are, on average, nearly indistinguishable. However, the spread about the mean of the observed $\left[A^{*}\right]^{\prime} s$ is appreciably larger than that of the simulated ones (respective variances are 0.14 and 0.02 about the fitted curves). While the nightly average in both panels is relatively constant for larger phase angles, it decreases sharply toward the full moon, implying that the earth is relatively more reflective at grazing angles (glint). Note that we have no reliable observations for phase angles below $40^{\circ}$ or above $140^{\circ}$ because of scattered moonshine and because of short observing periods, respectively.

The average albedo can be determined from our observations and simulations as $A=\frac{2}{3} \int_{-\pi}^{\pi} d \theta f_{L}(\theta) A^{*}(\theta)|\sin \theta|$. We have extrapolated $\left[A^{*}\right]$ from $40^{\circ} \leq|\theta| \leq 180^{\circ}$ to the full range of the integration using a constrained least squares fit in which $\left[A^{*}\right]$ is assumed to vary smoothly with lunar phase. This method is used widely and successfully in helioseismology [Goode, 1995]. We tested it by performing nearly 300 additional simulations, for nights other than the 143 between January 1999 and August 2000, so as to cover the entire range from $0^{\circ} \leq \theta \leq 180^{\circ}$ for the parts of the earth we "see" from BBSO. Fitting these and integrating $A^{*}$, we find

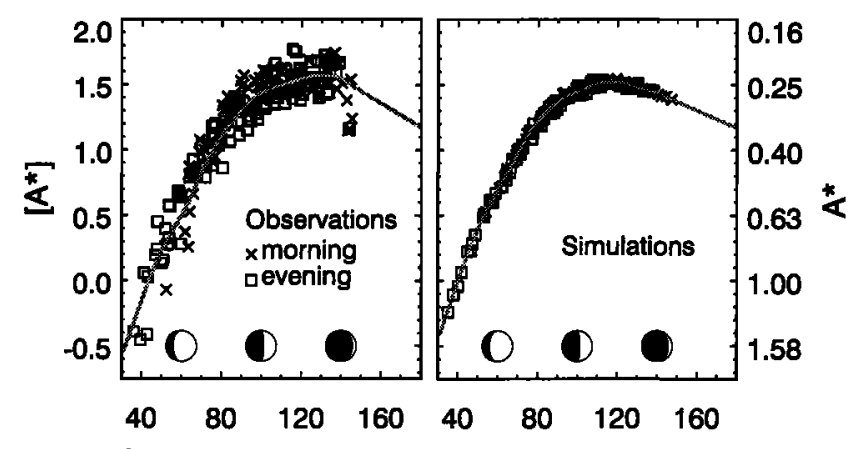

Lunar Phase (degrees) Lunar Phase (degrees)

Figure 1. Observed (left panel) $\left[A^{*}\right]^{\prime}$ s plotted against the absolute value of the lunar phase (also indicated by the cartoons along the $\mathrm{x}$-axis) from 198 clear observing nights from December 1998 through the beginning of September 2000. Simulations (right panel) are shown for the 143 nights for which there were also contemporaneous cloud cover data. Each data point represents a nightly average of 0.5-3.0 minute earthshine measurements taken once every five minutes with 0.1-5.0 second moonshine observations interspersed. Error bars would be within the symbols. Constrained least square fits to the data and simulations are shown by the curves. 


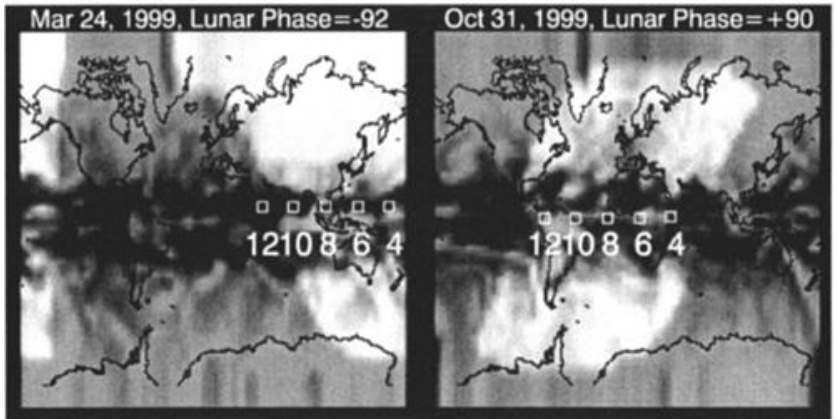



$242016 \quad 12 \quad 8 \quad 4 \quad 0$ Universal Time

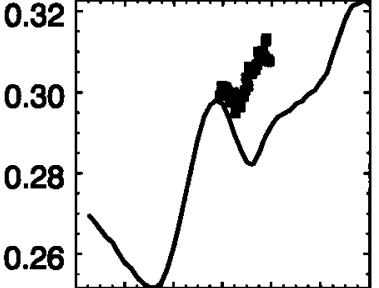

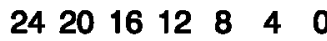
Universal Time
Figure 2. In the top panels, the bright areas highlight those parts of the earth that are the source of the earthshine (i.e., are simultaneously in the sunshine and are visible from the moon at some time during the observations); the satellite-derived WSI cloud cover data is shown in gray-scale, with brighter areas indicating greater cloud cover. For 31 October 1999, note that the northernmost regions are not sunlit and the southernmost regions do not contribute to earthshine because the moon is fairly far north in the sky. Simulations of $400+$ nights show that not detecting the pole in this way reduces $A$ by about 0.005 because of the nearly invariant relative brightness of the polar regions. The boxes indicate the longitudes of maximal contribution to the earthshine at the Universal Time (UT) shown. The solid boxes in the lower panels show the observed effective albedo as a function of time (note that the time axis is reversed), while the solid line indicates the simulated $A^{*}$.

$A=0.294$. We note that the fit to the full-range of simulations is indistinguishable from that shown in the right panel of Figure 1. In all cases, the extrapolation of $\left[A^{*}\right]$ for $|\theta|>140^{\circ}$ contributes about 0.07 to $A$, while the region for $|\theta|<40^{\circ}$ contributes about 0.01 . From this, we obtain $A=0.297 \pm 0.005$ for the observations (left panel) and $A=0.296 \pm 0.002$ for the simulations (right panel) for the two-thirds of the earth we observe. The uncertainties here arise from the large night-to-night and seasonal variations.

Our observations have also determined both the variation of the instantaneous $A^{*}$ during each night, and the variation of the nightly average $A^{*}$ throughout the seasons. In Figure 2 , we show one set of evening and one of morning observations near the waxing and waning quarter moon, respectively, as well as the corresponding simulations. The top panels show that a significant part of the earth contributes to the earthshine during the observations. For larger lunar phases, more of the earth contributes instantaneously to the earthshine, but the length of the observations is reduced. For smaller lunar phases, less of the earth contributes to the earthshine at any moment, but there is a relatively longer observing night. Hence, somewhat more than a third of the earth is covered during a given night, roughly independent of phase.

The observations are consistent with the simulations for 24 March 1999, which is one of the nights for which the agreement is quite good. On 31 October 1999, a more typical night, there is a discrepancy of about $5 \%$ relative (or 0.015 absolute) in the effective albedo. By scaling variations of the simulated $\left[A^{*}\right]$ 's to those of the simulated albedo, we estimate that measuring $\left[A^{*}\right]$ to an accuracy of 0.01 determines the global albedo, $A$, to 0.002 .

As the cloud cover is fairly high at geographical latitudes above $45^{\circ}$, the short timescale variations in reflectance are due to irregularities in the fractional cloud cover at low latitudes. In the left panel of Figure 2, one sees that the observed and calculated maxima in $A^{*}$ at 7:00 UT are due to a relative cloud excess over the Far East, while the calculated local minimum in $A^{*}$ at 11:00 UT arises from the cloudless area above India and the Arabian Sea. In the left panel, there is a nearly $10 \%$ change in $A^{*}$ through the period of the observations. An offset between the observed and calculated effective albedos is evident in the right panel; it typifies the general result that the observed night-to-night variations are greater than those of the simulations.

The seasonal variation of the earth's reflectance as determined from our earthshine observations is shown in Figure 3. As $\left[A^{*}\right]$ is a strong function of lunar phase, we consider the anomaly of each nightly average relative to the bestfit mean trend of all the observations. These anomalies, which are shown as fractional changes in $A^{*}$, carry information about weather, climate and surface type. There is the expected summer minimum, with fall and spring maxima, and the earth is brightest in the spring, when it is also the cloudiest.

The observed seasonal variation is correlated with the corresponding simulations. However, the observations show about twice as much variability, with the differences being greatest at the extremes. The seasonal variation of albedo is the origin of the relatively greater spread among the observed $\left[A^{*}\right]$ 's in Figure 1, and most likely, the offset between observations and simulations typified in the lower right panel of Figure 2. The muted seasonal amplitude of the simulations may well be associated with the coarse binning of the scene models and/or the use of simulated snow and ice cover.

Some sense of the longer term trend in the earth's reflectance can be had by comparing these data with 72 nights of earthshine observations taken from January 1994 through July 1995. (These latter data favor no particular season.) The average deviation of the nightly means of these data from the gray curve in the left panel of Fig. 1 translates to a $2.5 \% \pm 1.5 \%$ greater $A^{*}$. Most of the deviation is present in the morning data. The corresponding value is $0.0 \% \pm 0.9 \%$ for the January 1999 through July 2000 data. We note that 1994-1995 was during the most recent solar activity minimum, and that our result is consistent with the hypothesis, based on cloud cover data, that the earth's reflectance decreases with increasing solar activity [Svensmark and Friis- 


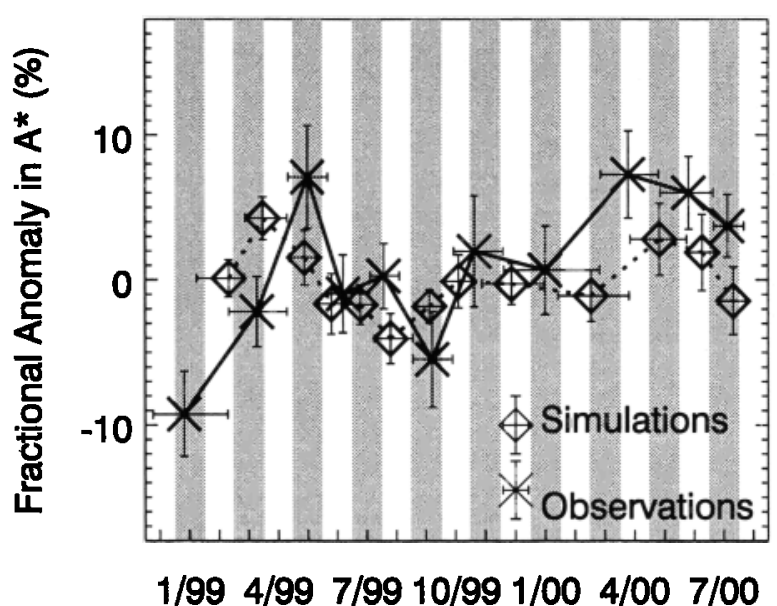

Figure 3. Seasonal anomalies in the effective albedo, $A^{*}$. From December 1998 through the beginning of August 2000, there are 170 nights of observations with 22 nights in the earliest bin, 23 nights in the next bin and 15 nights in each of the remaining 9 bins; the extra nights in the two earliest bins serve to reduce the uncertainties. From January 1999 onward, there are 143 nights for which we have both observations and contemporaneous satellite cloud cover data, which have been averaged in 13 bins with 11 nights in each. The $x$ 's show the mean of the observations, with the vertical bars being the standard deviation of the mean. The size of the latter stems from the large night-to-night variations in the cloud cover, rather than from uncertainties in the observations. The horizontal bars indicate the temporal span of each average. The diamonds indicate the corresponding simulation results. For the 143 nights for which we have observations and simulations, the anomalies relative to the separate fitted curves are correlated at the $95 \%$ confidence level.

Christensen, 1997]. On the other hand, the difference could be associated with the combined effect of the El Niño that occurred during the earlier observations and the La Niña (http://ww.ogp.noaa.gov/enso) during the latter.

Our demonstration that the earthshine can be observed with sufficient precision to derive meaningful information about the earth's optical reflectance suggests several further investigations. A multi-station network is now being established that will allow continuous global coverage during appropriate lunar phases. With the increased precision and coverage from the network, we will be able to monitor long-term variations in cloud cover and aerosols with sufficient accuracy to bear on climate change. Network observations of the reflectance in the coming years will also quantify ENSO events, volcanic eruptions, and decadal scale variability, including a more precise exploration of any solar cycle variation. Comparison of earthshine results with satellite observations (see, for example http://cloud.ucsd.edu/missions/triana.html) are also of clear interest to intercalibrate the methods and validate the scene models used.

Acknowledgments. We wish to thank those involved in earlier earthshine work at BBSO, especially J. Bruls, G. Eychaner, M. Haggerty, J. Maron and H. Zirin. This research was supported in part by grants from NASA (NAG5-7454) and the Western Regional Center of the National Institute for Global Environmental Change.

\section{References}

Danjon, A., Recherches sur la photométrie de la lumière cendrée et l'albedo de la terre, Ann. Obs. Strasbourg, 2, 165-180, 1928.

Danjon, A., Albedo, color, and polarization of the earth, in The Earth as a Planet, edited by G.P. Kuiper, pp.726-738, University of Chicago Press, 1954.

Dubois, J., Le photomètre à ceil-de-chat et ses applicatons, Ciel et Terre, 58, 350-361, 1942.

Dubois, J., Sur l'albedo de la terre, Bull. Astron., 19, 193-196, 1947.

Gibson, G., et al., Characteristics of the earth's radiation budget derived from the first year of data from the Earth Radiation Budget Experiment, SPIE, 1299, 253-256, 1990.

Flatté, S., Koonin, S.E., and MacDonald, G., Global Change and the Dark of the Moon, Report No. JSR-91-315, The MITRE Corporation, McLean, VA, 32 pp., 1992.

Goode, P.R., Internal Rotation and Structure of the Sun, ESA $S P-376,121-136,1995$.

Kondratev, K., in Radiation characteristics of the atmosphere and earth's surface, Translated from Russian by Vijay Pandit, General editor: V.S. Kothekar, Published for NASA by Amerind Pub. Co., New Delhi, 1973.

Rossow, W.B. and Lacis, A.A., J. Climate 3, 1204-1253, 1990.

Stephens, G., Campbell, G. and Vonder Haar, T., Earth radiation budget, J. Geophys. Res., 86, 9739-9760, 1981.

Suttles, J.T. et al., Angular radiation models for earthatmosphere systems. Vol I: shortwave radiation, NASA Reference Publication RP-1184, 26 pp., 1988. These scene models give local reflectance as a function of illumination and reflection directions, fractional cloud cover, and surface type.

Svensmark, H. and Friis-Christensen, E., Variation of cosmic ray flux and global cloud coverage - a missing link in solar-climate relationships, J. Atm. and Solar-Terrest. Phys., 59, 1225-1232, 1997.

P. R. Goode, J. Qiu, V. Yurchyshyn, and J. Hickey, Big Bear Solar Observatory, New Jersey Institute of Technology, Newark, NJ 07102. (e-mail: pgoode@bbso.njit.edu)

C. T. Brown and S. E. Koonin, W. K. Kellogg Radiation Laboratory, California Institute of Technology, Pasadena, CA 91125.

M-C. Chu, Department of Physics, The Chinese University of Hong Kong, Shatin N.T., Hong Kong.

E. Kolbe, Departement für Physik and Astronomie, Universität Basel, Basel, Switzerland.

(Received November 2, 2000; revised March 8, 2001; accepted March 14, 2001.) 